\title{
Wire Array Z-Pinch Insights for Enhanced X-Ray Production
}

T. W. L. Sanford, R. C. Mock, and R. B. Spielman Sandia National Laboratories, Albuquerque, NM 87185-1196

M. G. Haines and J. P. Chittenden

The Blackett Laboratory, Imperial College, London, SW7 2BZ, UK

\section{Prizentithe \\ JAN $21 \mathrm{ROS}$}

Q $8 \overline{T 1}$

K. G. Whitney and J. P. Apruzese

Naval Research Laboratory, Radiation Hydrodynamics Branch, Washington, DC 20375

\author{
D. L. Peterson \\ Los Alamos National Laboratory, Los Alamos, NM 87545
}

$\frac{1}{n}$

1

m

$Q$

$m$

N
J. B. Greenly and D. B. Sinars

Laboratory of Plasma Studies, Cornell Univerisity, Ithaca, NY 14853

D. B. Reisman

Lawrence Livermore National Laboratory, Livermore, CA 94550

D. Mosher

Naval Research Laboratory, Pulsed Power Physics Branch, Washington, DC 20375

PACS numbers: 52.35.Py, 52.55.Ez, 52.70.Kz. 52.70.La

*Sandia is a multiprogram laboratory operated by the Sandia Corp., a Lockheed Martin Company., for the US DOE under Contract DE-AC04-94AL8500 


\section{DISCLAIMER}

This report was prepared as an account of work sponsored by an agency of the United States Government. Neither the United States Government nor any agency thereof, nor any of their employees, make any warranty, express or implied, or assumes any legal liability or responsibility for the accuracy, completeness, or usefulness of any information, apparatus, product, or process disclosed, or represents that its use would not infringe privately owned rights. Reference herein to any specific commercial product, process, or service by trade name, trademark, manufacturer, or otherwise does not necessarily constitute or imply its endorsement, recommendation, or favoring by the United States Government or any agency thereof. The views and opinions of authors expressed herein do not necessarily state or reflect those of the United States Government or any agency thereof. 


\section{DISCLAIMER}

Portions of this document may be illegible in electronic image products. Images are produced from the best available original document. 


\begin{abstract}
Comparisons of measured total radiated $x$-ray power from annular wire-array $z$-pinches with a variety of models as a function of wire number, array mass, and load radius are reviewed. The data, which are comprehensive, have provided important insights into the features of wire-array dynamics that are critical for high $x$-ray power generation. Collectively, the comparisons of the data with the model calculations suggest that a number of underlying dynamical mechanisms involving cylindrical asymmetries and plasma instabilities contribute to the measured characteristics. For example, under the general assumption that the measured risetime of the totalradiated-power pulse is related to the thickness of the plasma shell formed on axis, the Heuristic Model [IEEE Trans. Plasma Sci., 26, 1275 (1998)] agrees with the measured risetime under a number of specific assumptions about the way the breakdown of the wires, the wire-plasma expansion, and the Rayleigh-Taylor instability in the r-z plane, interact. Likewise, in the high wire-number regime (where the wires are calculated to form a plasma shell prior to significant radial motion of the shell) the comparisons show that the variation in the power of the radiation generated as a function of load mass and array radius can be simulated by the 2-D Eulerianradiation-magnetohydrodynamics code (E-RMHC) [Phys. Plasmas 3, 368 (1996)], using a single random-density perturbation that seeds the Rayleigh-Taylor instability in the $r-z$ plane. For a given pulse-power generator, the comparisons suggest that (1) the smallest interwire gaps compatible with practical load construction and (2) the minimum implosion time consistent with the optimum required energy coupling of the generator to the load should produce the highest total-radiated-power levels.
\end{abstract}

\title{
INTRODUCTION
}

The discovery [1] that the use of very large numbers of wires enables high $\mathrm{x}$-ray power to be generated from annular wire-array z-pinches represents a major step forward $[2,3]$ in load design for large pulsed-power generators, and has permitted high $\mathrm{x}$-ray temperatures to be generated in radiation cavities on Saturn [4] and Z [5]. The implosion of a 300 tungsten-wire array within a 24-mm-dia.,10-mm-long cavity, whose outside wall forms the return-current return path on $Z$ by Porter [6], for example, has generated a brightness temperature of $145 \pm 10$ 
$\mathrm{eV}$. Such cavities are used to study radiation transport, opacity, and equation of state phenomenon in high energy-density plasmas. Importantly, the intense radiation generated from the high wire-number pinches is used to test radiation-drive geometries relevant to the ICF program $[7,8]$, such as in the static hohlraum concept of Olson $[9,10]$ or the dynamic hohlraum concept described in Ref. 10 by Nash, Derzon, Chandler et al. In this manuscript, changes in total radiated $\mathrm{x}$-ray power as a function of wire number [11], array mass [12], and load radius [13], generated from Saturn experiments with imploded 20-mm-long aluminum-wire arrays that led to these breakthrough hohlraum results are reviewed and contrasted with models and simulations. Because much of the wire-number analysis is new, this material is emphasized.

In the first section, the variation of Saturn aluminum data with wire-number is summarizied, showing that (1) the key parameter for producing high $\mathrm{x}$-ray power is the interwire gap spacing, and (2) values of this parameter to below $-0.5 \mathrm{~mm}$ are required for tight, wellbehaved pinches, and high radiated power. The two-dimensional (2-D) TIP [14] radiationmagnetohydrodynamics code (RMHC) simulations with various wire numbers [15] are then reviewed and contrasted with two state-of-the-art RMHCs, MH2D [16] and MH3D [17] that calculate the evolution from the solid to plasma state of individual wires in the $r$ direction and in the r-z plane, or for multiple wires in the $x-y$ plane, respectively. They use LTE ionization, Thomas-Fermi EOS, and Lee and More resistivity and thermal transport $[18,19]$. Calculations . from these codes are compared with the plasma formed from a single wire on the Cornell $\mathrm{x}$-pinch pulser [20] using a prepulse current similar to that flowing on Saturn [1]. The plasma is measured with an $\mathrm{x}$-pinch backlighter [21]. This comparison provides qualitative confirmation of the calculations at the end of the current prepulse phase. In this section, the evolution of the wire plasmas and their subsequent merger into an annular plasma shell prior to stagnation as a 
function of wire number is connected to the explosion dynamics of the arrays, using the recently developed Heuristic Model [22].

In the second section, the measured radiation and pinch-plasma characteristics for the high wire-number implosions, which generate the highest peak total $\mathrm{x}$-ray powers, are briefly summarized. The data show that the narrow ( $<5 \mathrm{~ns}$ ) radiation pulses generated by interwire gaps below $\sim 0.5 \mathrm{~mm}$ can be realistically simulated by the 2-D Eulerian radiationmagnetohydrodynamic code (E-RMHC) [23, 24], in which Rayleigh-Taylor (RT) growth dominates the implosion dynamics in the $\mathrm{r}-\mathrm{z}$ plane. For these simulations, the array is idealized as a uniform, cylindrically-symmetric shell with a random-density variation in the r-z plane that seeds the RT instability.

In the third section, the total $\mathrm{x}$-ray pulsewidth and peak power as a function of array mass and load radius for these high wire-number loads are discussed. The E-RMHC simulations give reasonable agreement with the total radiated power data over an order-of-magnitude variation in array mass and almost a factor of three change in load radius. The simulations suggest that in $2-$ $\mathrm{D}$, the RT growth occurs via a two-staged process [24] which limits the RT development, and thus permits high $\mathrm{x}$-ray power to be generated. The simulations also show the benefit to the generation of high radiation powers of using low-enough mass arrays and small-enough radius loads to minimize the growth of the RT instability balanced against the need for a high implosion velocity.

In each section, the measurements are compared with either analytical or numerical simulations in 1-D or 2-D in order to connect features of the underlying dynamics of the implosion with the observed $\mathrm{x}$-ray emission characteristics. The emission characteristics seen on the Saturn aluminum experiments are also compared with a few related characteristics of high 
wire-number tungsten arrays measured on $Z$. The measurement comparisons with the Heuristic Model and E-RMHC simulations provide support for the underlying theoretical assumptions, and the ability of the model and code to provide an interpretation of the $\mathrm{x}$-ray performance with wire number, array mass, and array radius.

The geometry and experimental arrangement of the Saturn and $\mathrm{Z}$ measurements are discussed in Refs. 1, 11, 12, 13, 25 and Ref. 5, respectively.

\section{WIRE-NUMBER VARIATION}

\section{A. Measurements}

In the first set of Saturn aluminum wire-number experiments $[1,11]$, the array mass was fixed and the initial interwire gap was varied by more than an order of magnitude from 6 to 0.4 $\mathrm{mm}$ by simultaneously changing the number of wires (from 10 to 192) and the wire diameter. This procedure was carried out for $0.62-\mathrm{mg}$ and a $0.84-\mathrm{mg}$ arrays having initial radii of $8.6 \mathrm{~mm}$ and $12 \mathrm{~mm}$, respectively. Decreasing the interwire gap resulted in monotonic decreases in the risetime and width of the $\mathrm{x}$-ray pulse and simultaneous increases in total radiated power and energy, in the same way for both array radii (Figure 1). Over the 6 to $0.4 \mathrm{~mm}$ gap reduction explored, the total radiated power increased by a factor of 20 (Figure 1A) and the total radiated energy by a factor of 2 (Figure 1C).

In addition, for gaps smaller than $2 \pm 0.6 \mathrm{~mm}$, the character of the $\mathrm{x}$-ray emission qualitatively changed, from a broad, irregular radiation pulse at large gaps (Fig. 2A) to a narrow, evenly-shaped radiation pulse that was followed by a much weaker pulse at small gaps (Fig 2B). In the Fig. 2, the $\mathrm{x}$-ray pulses measured in the energy channels $0.2-2,2-3$, and $4-6 \mathrm{keV}$ are shown, normalizied to the peak value measured at the first radiation peak. The weaker second 
radiation peak is consistent with a second radial implosion and corresponds to a pinch having a lower temperature relative to that of the first implosion (Sec. II). For gaps greater than $\sim 2 \mathrm{~mm}$, time-integrated images of the pinch (mainly showing the plasma at stagnation) exhibit the presence of a kink $(m=1)$ as well as a sausage $(m=0)$ instability (Fig. 2 C). Time-dependent images show significant precursor plasma stagnating on axis, generating soft $\mathrm{x}$-ray emission tens of nanoseconds prior to the main implosion, in agreement with earlier [26] and current [27] experiments. For gaps less than $\sim 2 \mathrm{~mm}$, in contrast, no kink instability is observed, with only a minimal precursor plasma forming (Fig. 2D). Moreover, the changes with gap in the temporal shape of the $\mathrm{x}$-ray pulse (Figs. 2A and 2B) and in the spatial quality of the pinch (Figs. $2 \mathrm{C}$ and 2D) occurred with corresponding quantitative transitions in the rates of change of the emitted total $\mathrm{x}$-ray power (Fig.1A), the average size of the $\mathrm{K}$-shell emission region (Fig. 1B), and the emitted total $\mathrm{x}$-ray energy (Fig. 1C). Corresponding variations in the average $\mathrm{K}$-shell emitting ion density and electron temperature were also observed [11]. These density and temperature measurements show that the increase in $K$-shell power (and by inference the total power) is the result of systematically greater plasma compression and is not the result of an increase in temperature.

Not enough data were taken at wide gap spacings, however, to ascertain how sharply this transition in $\mathrm{x}$-ray behavior took place. For this reason, we represent it hypothetically as a transition between two power laws as illustrated in Fig. 1, with the gap exponents indicated. In Fig. 1C, the measured total radiated energy is normalizied to the calculated 0-D kinetic energy for a 10-to-1 radial convergence. The change in this normalizied energy (Fig. 1C) is approximately consistent with the change in a calculated 0-D kinetic energy (dotted lines in Fig. 1C) now evaluated from the experimental radial convergence (Fig. 1B). 
The greater rate of change of the pulsewidth with gap for gaps greater than $\sim 2 \mathrm{~mm}$ (Fig. 1A) reflects the greater disorganization of the implosion as seen by the lost double-pulse nature of the stagnation (Fig. 2). If instead of the pulsewidth of the total power pulse, the pulsewidth of a harder $\mathrm{x}$-ray detector channel (which is insensitive to the radiation from the second implosion owing to the reduced temperature of the implosion [sec. IIA]) is plotted, the pulsewidth shows no discontinuity with gap, as illustrated in Fig. ID for the 4-6 keV channel. Moreover, the greater rate of decrease in power for large gaps relative to that for small gaps (Fig. 1A) is consistent with the decrease in total radiated energy (Fig. 1C). Specifically, in Fig. 1D, the peak total power of Fig. 1A normalizied by the energy of Fig. IC shows no discontinuity at $-2 \mathrm{~mm}$, and scales like $\mathrm{G}^{-0.93 \pm 0.1}$ over the entire range measured. The variation in peak total power so normalizied now tracks the inverse of the pulsewidth (albeit for a pulsewidth corresponding to the higher energy $x$-ray band), which scales as the gap to the $0.81 \pm 0.1$ power (Fig. 1D), as would be expected when the total energy radiated during stagnation is slowly varying.

\section{B. Modeling}

\section{TIP Simulations}

The apparent transition in implosion quality near the 2-mm gap has been interpreted using the 2-D TIP RMHC in $x-y$ geometry, using 1-D numerical simulations of the individual wire explosion from a cold start as input [1]. Prior to wire-plasma merger in the array, the density distribution from each wire was characteristic of a Gaussian having a $\sim \mathrm{I} \mathrm{VV}$ temperature. Calculations performed with these codes show (in correspondence with the experimental data) that a change in the implosion topology occurs with increasing wire number. The calculated implosion is seen to make a transition from one composed of non-merging, selfpinching, individual wire plasmas to one characterized by the early formation and subsequent 
implosion of a quasi plasma shell. The shell has density and current variations distributed azimuthally that are correlated with the initial wire location and which decrease in amplitude with decreasing gap. In the Heuistic Model [Sec. B3] in contrast, the individual wires are assumed to be $m=0$ unstable and always expanding (as in single wire experiments [28]) though the necking regions are pinching; the transition in implosion quality is associated with the merging occuring before or after significant inward motion of the array. Another physical possibility for the transition could be the significantly larger inward radial streaming from coarse arrays [27].

The calculated transition region is sensitive to (1) the magnitude of the prepulse that accompanies the main current pulse, (2) the current flowing per wire, (3) the interwire gap, and (4) the resistivity model used. For the particular resistivity model used and for the measured prepulse and wire sizes used, this transition was calculated in 2-D to occur between wire numbers 20 to 80 (or between interwire gaps of about 3 to $1 \mathrm{~mm}$, respectively) for the small radius load [1]. For wire number $(\mathrm{N})$ greater than 80 , the calculated individual wire plasmas in 1D never self-pinched, because the current per wire was too low. For $\mathrm{N}$ less than $\sim 20$, the current per wire was high enough that the calculated individual wire plasmas always self-pinched in 1-D before stagnation occured. For $N \sim 40$, the calculated wire plasmas in both 1-D and 2-D partially merged prior to significant radial motion of the array. For descriptive purposes, we thus refer to the small wire-number region where the gap is greater than $2 \mathrm{~mm}$ as the "wire-plasma" regime, and the high wire-number region where the gap is less than $2 \mathrm{~mm}$ as the "plasma-shell" regime, knowing that in this regime there can still be significant azimuthal density variations.

The calculated transition was also seen to be consistent with observations made with 1.3mm gap loads in the transition region on Saturn, as is illustrated in Fig. 3 [11]. In Fig. 3B, 
individual wire plasmas are observed to neck-off in the form of bright spots (similar to Beg et al. $[28,29]) 20$-ns prior to peak radiated power (Fig. 3C), when the array has only imploded a fraction of a mm radially (as shown by the dashed curve in Fig. 3A). Ten nanoseconds later, after the array has imploded an additional $1.5 \mathrm{~mm}$, the observed plasma emission becomes a continuous distribution, with no evidence of individual wire structure, suggestive of plasma merger.

The TIP $x-y$ simulations [15] together with analytic wire modeling [30] show that the wire plasmas (in contrast to the plasma of a shell) may accrue azimuthal velocity components during the implosion owing to (1) deviations in the locations of the individual wires from those of a perfect annulus, or (2) to wire-to-wire current nonuniformities, or (3) to the presence of the limited number of current return posts [31] surrounding the array, which could seed flute-mode instabilities. These velocity components in a plasma-wire regime implosion can produce increased density asymmetries at stagnation relative to that of an imploding plasma-shell implosion. The resulting difference in the calculated radial convergence between the implosion of a load in the wire-plasma regime relative to that of a load in the plasma-shell regime is illustrated in Figs. 4A and 4B, respectively, for a 10 current-return-post geometry as used in the experiments. In the Fig. 4 simulation, the variation in initial wire position arises from the numerical noise. Such asymmetries contribute to the reduction in both the compressibility of the stagnating plasma and the resulting radiated energy, both in qualitative agreement with the discontinuity observed in the radial convergence measurements (Fig. 1B) and in the energy channel (Fig. 1C). Illustrated also in Fig. 4 is the effect that the 10 current-return posts have on the azimuthal distribution at stagnation. The imprint of such an azimuthal structure has been observed from the radiation field generated from foam targets placed interior to the wire array by 
Derzon et al. [32]. Nash et al. [33] have shown by replacing the posts with an azimuthally continuous shell that the on-axis radiation generated from a dynamic hohlraum configuration is doubled, again illustrating the importance of maintaining azimuthal symmetry throughout the entire load region for producing high radiation yields.

The TIP simulations in the $x-y$ plane [15] show, however, that variations in wire number or other azimuthal asymmetries cannot account for the measured order-of-magnitude change in $\mathrm{x}$-ray power pulseshape (Figs. 1D, 2A, and 2B) [15]. In contrast, TIP [15] and E-RMHC [11] simulations suggest that the measured change in peak $\mathrm{x}$-ray power (aside from the topologychange effect) with gap (Fig. ID) are related to the evolution of the thickness of the plasma shell due the growth of RT instabilities in the r-z plane, as is now discussed.

\section{MH2D and MH3D Simulations}

Before the detailed numerical and analytic modeling with wire number is presented for the small-radius $(8.6 \mathrm{~mm}$ ) geometry using $\mathrm{MH} 2 \mathrm{D}$ and $\mathrm{MH} 3 \mathrm{D}$, however, the expansion of a single aluminum wire in that geometry, resulting from the measured Saturn current pulse, is described. The measured Saturn current consists of a prepulse that approximately ramps from 0 to $100 \mathrm{kA}$ in $100 \mathrm{~ns}$ prior to the start of the main pulse, and a main pulse that rises to a peak current of $\sim 7 \mathrm{MA}$ in $\sim 35 \mathrm{~ns}$. The earlier [1] and recent simulations discussed here indicate that the wire-plasma expansion in the prepulse phase affects the merger of the wire-plasmas and resulting shell structure. Moreover, the TIP $x-y$ simulations suggest that the character of a singlewire plasmas calculated in 1-D provides a qualitative indicator of the behavior of a wire plasma within an array [15], with regard to self pinching or not in the $x-y$ plane (as is again borne out 
with the following improved resistivity simulations). Shown in the next section, the timing of the merger affects the resulting RT seed and the subsequent implosion dynamics.

Figure $5 \mathrm{~A}$ illustrates the calculated mass density of the aluminum wire plasma just after the end of the prepulse, starting from the solid phase, using MH2D run in the r coordinate only. Figure 5B depicts the associated calculated ion temperature, electron temperature, and ionization state. The wire is characteristic of one of the $15-\mu \mathrm{m}$ diameter wires in an array of 64 wires. At this time, $98 \%$ of the wire mass is calculated to remain in a high-density, cold core, which has expanded to a radius of $\sim 100 \mu \mathrm{m}$. Despite the presence of a conducting wire core, however, $90 \%$ of the current flows in the surrounding low-density plasma (corona) due to the larger crosssectional area and lower resistivity. The corona extends to a radius of several $\mathrm{mm}$, has an ion temperature of $\sim 1 \mathrm{eV}$, and an electron temperature of $\sim 1$ to $12 \mathrm{eV}$ that increases with radius (Fig. 5B). Trac-II $[35,36]$ simulations of this geometry give qualitatively similar results. These results are in contrast to the earlier 1-D simulations [1] used as input to TIP, which showed a relatively hot core at this time. The difference arises from the approximate resistivity model used in the 1-D simulations [37].

Figure $5 \mathrm{~A}$ also shows the line-integrated mass-density profile for a $13-\mu \mathrm{m}$ diameter aluminum wire measured under these same end-of-prepulse conditions using the Cornell $x$-pinch backlighting source [20]. The technique employed was $\mathrm{x}$-ray shadowgraphy, which had less than 5- $\mu \mathrm{m}$ and $1-\mathrm{ns}$ resolution [21]. The two measured line-integrated mass profiles (denoted by 1 and 2) shown in Fig. 5A from the same wire are characteristic of the range of variation seen along wires, for repeated shots. The profiles confirm the presence of an expanding dense core, with a sharply defined edge at a radius that is within the range of the above MD2D simulations. The prepulse, which takes the wire through the verycomplex initial stage of explosion and up to 
an order of magnitude expansion of the high-density core, which contains almost all the mass, is a good first benchmark for the codes.

In the 1-D MH2D simulation of Fig. 5A, the radial extent of the corona is well past the half-gap (G/2) spacing of $0.42 \mathrm{~mm}$, suggesting the beginning of plasma merger if the wire was present in the array of 64 wires. Figure 6 plots the associated MD2D calculated electron-number density in 1-D at a radius of $\mathrm{G} / 2$ as a function of time (for such single-wire plasmas), assuming that the wire would be an element of a $16,24,48,64$, or 136 wire array. Throughout the prepulse, the density increases for all the wire-number configurations. The rapid increase in density at $0 \mathrm{~ns}$ is associated with the onset of the main current pulse. As in the earlier 1-D simulations [1], for the higher wire-number configurations $(N \geq 64)$, the magnetic field is too weak to cause self pinching of the indiviual wire plasma, and the density continues to increase. For the lower wire-number configuration $(N \leq 48)$, the density continues to rise and then falls, as the wire plasma is swept up by the stronger field and then is returned to the wire axis. The transition between self pinching and continued expansion in 1-D thus occurs between $\sim 48$ and $~$ 64 wires. It is consistent with Trac-II and the earlier simulations [1], suggesting that the breakpoint in topology as calculated in 1-D (within the limited-geometries simulated) is somewhat insensitive to the details of the particular resistivity model used.

2-D MH2D simulations in the $r-z$ plane of a single wire (from a cold start) modify these results slightly, as shown in Figs. $7 \mathrm{~A}$ and $7 \mathrm{~B}$ for one of $24,25-\mathrm{mm}$ and one of $64,15-\mathrm{mm}$ wires $30 \mathrm{~ns}$ into the main current pulse, respectively. Such simulations show that on average that the self-pinching only occurs in the necks of instabilities and the remainder of the corona hardly pinches at all. The $m=0$ instabilities set in just when in 1-D the magnetic field would be large enough to pinch the coronal plasma. As a result, there is continued expansion of the plasma in 
the bulges, while pinching only occurs in the necks, as shown by Beg et al [28] in single-wire experiments. On average though, the self-pinching, which is calculated in 1-D represents the average trajectory of the surface simulated in 2-D. Importantly, these 2-D simulations show that due to the higher current per wire for wires in a lower wire-number array, the instability development is much greater, which leeds to more dramatic self-pinching that exacerbates the $\mathrm{m}=0$ development.

Figures $7 \mathrm{C}$ and $7 \mathrm{C}$ illustrate the modification to the single-wire density distributions, now in the $x-y$ plane, 41-ns into the main current pulse, using MH3D run in a 2-D mode, when the wires are placed in an annulus, for a 16- and a 48-wire array, respectively. In MH3D, cold, solid wires are used as the initial conditions. Both configurations exhibit the low-density corona being swept around the dense cores by the global magnetic field, which streams towards the axis, forming a precursor plasma. With the 16-wire array, the plasmas have self-pinched, in agreement with the single-wire simulations. With the 48-wire array, the low-density regions have merged, but the higher density regions still retain the characteristically tear-drop shaped azimuthal asymmerty, again in qualitative agreement with the 1-D simulations (Fig. 6). The global field distorts the otherwise symmetrical expansion of the wire plasma into the characteristic tear-drop shape shown. The precursor plasma is more pronounced owing to the deeper radial penetration of the global magnetic field between wires with decreased wire number, as is observed experimentally (Figs. 2A and 2B). Because of the hotter individual wire plasma calculated in the 1-D simulations that were used as input to TIP (Sec. IB 1), the earlier TIP simulations [1] did not exhibit the tear-drop shape as is now calculated.

\section{Heuristic Model Calculations}


The Heuristic Model integrates the above simulations (Figs. 7A and 7B) and the experimentally measured [28] $\mathrm{m}=0 \mathrm{MHD}$ instabilities, observed in a single wire, into the seed for the RT instability. The level of MHD instabilities reached at the time of merger is greater with arrays of small wire number due to the increased current per wire and the longer time before merger occurs. It is the development of this MHD sausage instability, at the time the wire plasmas from neighboring wires have merged, that is assumed to generate the seed of the RT instability in the Heuristic Model.

Specifically, the model is based, in part, on the following assumptions. The plasma corona in each wire expands with a mean velocity taken from the experiment of Beg et al. [28], while the MHD $m=0$ sausage instabilities develop non-linearly on a time scaling with the ratio of the instantaneous plasma radius to the Alfven speed, the time-scale again being fixed by the experimental data [28]. By the time of merger the global RT instability has a faster growth rate, and from then on this is assumed to be dominant. The wire-to-wire sausage perturbations are uncorrelated. At the merging time, the mean radius of the individual wire plasma is half of the interwire gap, so that the thickness of the annulus at merging is given by the gap. A statistical average in perturbed line density over all the wires in the array is then used to determine the initial axial perturbation. Analytic estimates are then made of its effect on the development of RT instabilities, linear and non-linear, during implosion of the annulus and subsequent radiation characteristics. The dependence of implosion quality on gap enters (1) in the thickness of the initial annulus and a reduced average perturbation amplitude for larger wire numbers, (2) in the degree of radial plasma jetting (Figs. 7C and 7D) taken from the experiment of Lebedev et al. [27], and (3) in an effect of slower energy equipartition between ions and electrons estimated for long equipartition times. The dominant wavelength of the $\mathrm{RT}$ is found at stagnation. 
Moreover, in the Heuristic Model the geometrical connection between the array radius $\left(a_{m}\right)$ at which merger occurs and the trajectories of the wire plasmas up to this merger time $\left(t_{m}\right)$ lead to a very nonlinear relation for $\mathrm{t}_{\mathrm{m}}$ and $\mathrm{a}_{\mathrm{m}}$ and to a division of behavior above and below $\mathrm{a}$ critical wire number $\left(\mathrm{N}_{\mathrm{c}}\right)$ or gap $\left(\mathrm{G}_{\mathrm{c}}\right)$ (Fig. 8A). The critical wire number or gap is directly related to the transition discussed in Sec. IA. Namely, for $\mathrm{N}<\mathrm{N}_{\mathrm{c}}$, the wires spend the bulk of their implosion time in a wire-plasma regime, and for $N>N_{c}$, the wire plasmas have merged before significant motion of the array has occurred. Figure $8 \mathrm{~A}$ shows the ratio of $\mathrm{t}_{\mathrm{m}}$ to the time it takes for the array to pinch $\left(t_{p}\right)$, and the ratio of $a_{m}$ to the initial radius $\left(r_{0}\right)$ as a function of gap, again for the small-radius load of $0.86 \mathrm{~mm}$. For this Saturn geometry, $t_{p}=60 \mathrm{~ns}$, and $\mathrm{N}_{\mathrm{c}}=35$ wires with a corresponding $G_{c}=1.6 \mathrm{~mm}$, in agreement with the above numerical simulations and measurements of Sec. IA.

Figure $8 \mathrm{~B}$ shows that the measured risetime of the total radiated power pulse as a function of gap is well described by the model, assuming a linear relation between the plasma shell thickness on axis and the risetime. In the figure, the various components of the model are displayed. There, $\Delta_{\mathrm{m}}$ represents the contribution arising from the shell thickness at merger, whereas $\delta_{\mathrm{a}}$ represents the contribution arising from the increased thickness due to the growth of the RT instability after merger, with the initial-seed perturbation determined by the uncorrelated $m=0$ instabilities on individual wires. Sum corresponds to the sum of the two terms. The inclusion of the measured jetting, arising from the effect of the global field, leads to a greater thickness of the shell at merger, as indicated by the evolution to the ellipticity curve. The effect 
of the slower equipartition of energy from ions to electrons to $\mathrm{x}$-rays is triggered at large gaps, because the thickness at merger dominates, causing the final pinch radius to be large, the number density to be low, and the load current to be large (smaller inductance). It, in combination with the sum of all the contributions, is indicated by the highest curve. This final addition has a large uncertainty at the larger gaps owing to the magnitude of the measured load current as a function of gap used in the model and to the actual size of the pinch. Increasing the measured current by $10 \%$ increases this equipartition contribution by $50 \%$, whereas reducing the calculated pinch size by a factor of two reduces the contribution by $50 \%$, as is illustrated by the single error bar on the model at the largest gap.

For very small gaps, the modeled risetime scales as $G^{0.5}$, which results from the nonlinear growth of the RT instability in $\delta_{2}$. This variation is nicely illustrated when the number of wires is doubled from 120 to 240 on $\mathrm{Z}$, for the small-radius geometry using tungsten wires [37]. On $\mathrm{Z}$ the total radiated power increased by $(45 \pm 22) \%$ and the pulsewidth decreased by (29 \pm 9$) \%$, in agreement with the $41 \%$ change expected from the model.

\section{Other Models}

As is illustrated by the Heuristic Model in its comparison with the data of Fig. $8 \mathrm{~B}$, the simple measured scaling of the risetime with gap shown in Fig. 8B (as well as in Fig. 2E) is likely masking several complex procèsses. A variation of the Heuristic Model makes a different assumption for the gap dependence of the annular perturbation formed by the merged wires that may help to clarify some of these processes [38]. Gamble II 10-tungsten-wire linear-array experiments at the Naval Research Laboratory show bright-field Schlieren images of short-axialwavelength plasma flares around expanding wire-plasma cores that are remarkably similar to aluminum-wire observations [28] and MH2D carbon-fiber simulations [39] (Fig. 9). In these 
works, sausage perturbations growing on the expanding wire plasmas are nonlinear and largeamplitude even at very-early times in their development. The shapes of the perturbations are close to self-similar in the radial scale, that is, they "look" the same independent of radius. Thus, the fundamental (longest) wavelength in the perturbation spectrum grows as the wire plasma expands, so that at the merging time, both the thickness of the annulus and the dominant perturbation wavelength are comparable to the interwire gap. The gap dependence of implosion quality and radiated pulse width then derives from larger gaps (smaller wire numbers) producing longer-wavelength perturbations that are more disruptive during implosion of the annulus. For this Self-Similar Model, the amplitude of the initial annular perturbation is always large and the wavelength spectrum varies with gap, while in the Heuristic Model, the perturbation amplitude varies statistically with wire number.

In contrast to either of these two models, another interpretation [38] of the measured pulsewidth is provided by the analytic KART model of Desjarlais and Marder [34]. This model is based on the enhancement of a global RT instability that arises from the kink instability of the individual wires, which acts in phase with the RT instability. The model is discussed in detail in Ref. 34.

\section{CHARACTERISTICS AT HIGH WIRE NUMBER}

\section{A. Measurements}

The aluminum loads on Saturn in the plasma-shell regime having interwire spacings of less than $\sim 0.6 \mathrm{~mm}$ exhibit both a strong first and weaker second radiation pulse (Figs. 2B and 10A) that correlate in time with a strong and then weak measured radial convergence (Fig. 10B), hotter and then cooler electron temperatures of the radiating core (Fig. 10C), and higher and 
then lower ion velocities of the emitting plasma (Fig.10D), as expected for a double implosion [25]. Images of the $\mathrm{x}$-ray emission from the imploding shell a few nanoseconds prior to the first implosion exhibit the classic bubble and spike structure associated with the RT instability. Axial lineouts of the images in the $r-z$ plane show that the wavelength of the RT structure is in the range of 1 to $3 \mathrm{~mm}$, in agreement with RMHC simulations $[15,24,40]$ and the Heuristic Model.

\section{B. Modeling}

Two-dimensional RMHC simulations $[23,40]$ in the $[-z$ plane reproduce a number of the characteristics exhibited in Fig. 10 for these azimuthally-symmetric arrays, as is illustrated by the E-RMHC simulation of the pulseshape and spatial size shown in Figs. 10A and 10B, respectively. For such simulations, the magnitude of the random-density perturbation in a given initial plasma-shell thickness is adjusted to give the measured pulsewidth at stagnation. This perturbation seeds the growth of the RT instability, which limits the radial convergence in the $r-z$ plane. For the aluminum E-RMHC simulations discussed here, the emission opacity (electronphoton coupling) approximation used, however, allows greater radiation cooling to occur than is physical. The cooler plasma has reduced radial expansion following stagnation and the simulated plasma rapidly reaches a quasi-equilibrium without going through the second compression seen experimentally (Fig. 10). This is not the case for tungsten implosions, where the multitude of final states in tungsten relative to aluminum permits the plasma to rapidly cool and only a single implosion is typically seen $[5,23]$. A reduction in the plasma electron-photon coupling constant (denoted by E-RMHC:R in the figures), as is done in some of the E-RMHC simulations (to account for the reduced emission from aluminum), nevertheless, permits more realistic peak powers to be simulated at low mass (Sec. III). These simulations simultaneously generate a second quasi-convergence as shown in Figs. 10A and 10B. The use of the nominal electron- 
photon coupling in the E-RMHC simulations is denoted by E-RMHC:N. Radiation pulseshapes at the first peak, however, using either coupling agree with one another [12], and thus indicate that (aside from the double bounce, which is sensitive to the coupling) the dynamics creating the pulseshape of the first pulse are relatively independent of the radiation emission, in these simulations.

\section{MASS AND RADIUS VARIATION AT HIGH WIRE NUMBER}

\section{A. Measurements}

Following the wire-number scan discussed in Sec. IA, two additional series of Saturn aluminum-wire experiments were conducted in the calculated high wire-number, plasma-shell regime. These experiments were designed to better understand the conditions that optimize the total radiated power in this regime [41]. In the first series, the radius was held fixed at $12 \mathrm{~mm}$ and the mass was varied over the range 0.42 to $3.4 \mathrm{mg}$ [12]. This series showed that when the mass of the array (which used $~ 192$ wires) is reduced from above 1.9 to below $1.3 \mathrm{mg}$, a factor of two decrease in pulsewidth (Fig. 11A), with an associated doubling of the peak total-radiatedpower, (Fig. 11B) occurs. The variation observed is qualitatively similar to that recently measured on $Z$ [5, 37], for tungsten-wire arrays, using 120 wires at a radius of $20 \mathrm{~mm}$ (Fig. 11C and 11D). In the second series, the array mass was held fixed, near a value which maximizied the peak power from the first series, and the array radius was varied [13]. This series showed that when the radius is increased from 8.6 to $20 \mathrm{~mm}$, for a mass of $0.6 \mathrm{mg}$ in 136 wires, the total radiated pulsewidth (Fig. 11E) increases from only $\sim 4$ to $\sim 7$ ns and the associated peak total radiated power (Fig. $11 \mathrm{~F}$ ) remains relatively unchanged with radius. This result suggests that the quality of the large-radius implosions provided by the large number of wires in the initial array 
reduces the disruptive effects of the RT instability seeding previously observed in small wirenumber, large-radius loads [42].

\section{B. Modeling}

The E-RMHC simulations show that over the mass- and radius-range measured, which spanned an implosion time of 40 to $90 \mathrm{~ns}$, the shape of the simulated total-radiated-power pulse tracks the initial pulse measured $[12,13]$. Moreover, the implosion time of the simulated pulse agrees with that measured, within a shot-to-shot variation of only $2 \mathrm{~ns}$. This agreement suggests that $(100 \pm 7) \%$ of the initial mass is being accelerated during the implosion, for these high-wire -number loads. Within the uncertainty of the electron-photon coupling approximation, the measured pulsewidth (Figs. 12A and 12E) and trends in total radiated peak power (Fig. 12C and 12E) reasonably track with those simulated, using only a single value of a density perturbation seed. The qualitative agreement in these comparisons that span an order-of-magnitude in load mass and almost a factor of 3 in array radius and that use this single-seed value come about in calculations in which a two-staged RT growth occurs in the E-RMHC simulations. This result also suggests that because in the radius scan the gap varied from 0.4 to $0.96 \mathrm{~mm}$ and because the 2-D simulation does not know about the discrete azimuthal nature of the array, the fact that the single seed describes the data implies that other asymmetries ( $\mathrm{Sec}$. IB1) may be beginning to dominate these high wire-number implosions.

Importantly, these simulations attribute the decrease in pulsewidth and associated doubling of the peak total power, as mass is reduced, to the faster implosion velocity of the plasma shell relative to the growth of the shell thickness (Fig. 12A). The similarity of the pulsewidth and peak power variation with mass of the $Z$ data with tungsten (Figs. 11C and 11D) 
with that of the Saturn data with aluminum (Figs. 11A and 11B) suggest that the same dynamics that is behind the Saturn data is also behind the $\mathrm{Z}$ data. The simulations also correlate the decrease in pulsewidth with radius (Fig. 11E) to the faster decrease of the shell thickness relative to the decrease in shell velocity (Fig. 12B).

\section{CONCLUSIONS}

Implosions that develop narrow $x$-ray pulsewidths with high peak powers can be generated from both small- and large-radius annular wire arrays by keeping the interwire gap to spacings on the order of $0.5 \mathrm{~mm}$ or less for Saturn and $\mathrm{Z}$ pulsed-power conditions. Reducing the implosion time in these experiments (by decreasing either the mass or radius), led to the highest powers being developed, which, in the modeling, corresponded to a reduction in the growth of the axial RT instability relative to the implosion velocity. The Heuristic Model and the E-RMHC simulations agree with aspects of the data and help to quantify the role of plasma asymmetries and instabilities on the underlying dynamics. Whilst we have found empirical scaling relations and have developed models and simulations that quantitatively track the measurements, more detailed measurements and more sophisticated modeling are required to verify some of the conjectures made. In conclusion, the experiments and analyses discussed here conclusively demonstrate the necessity of using a large number of wires within a wire array in order to optimize the total $x$-ray power from and energy coupling to $z$-pinch implosions. Their utilization has (1) enabled the attainment of high-power in load configurations for large pulsed-power $\mathrm{x}$-ray generators and (2) permitted a better understanding of the dynamics responsible for the high radiation powers generated to be made. 


\section{ACKNOWLEDGMENTS}

We thank the late G. O. Allshouse, C. Deeney, M. P. Desjarlais, J. S. DeGroot (UCD), T. A. Haill, D. A. Hammer (Cornell), J. H. Hammer (LLNL), B. M. Marder, Y. Maron (Weizmann Institute), M. K. Matzen, T. J. Nash, P. E. Pulsifer (NRL), K. W. Struve, W. A. Stygar, J. W. Thornhill (NRL), A. Toor (LLNL), and F. J. Wessel (UCI) for insightful technical discussions; S.A. Pikuz and T.A. Shelkovenko for development of the x-pinch backlighter; G. A. Chandler, D. L. Fehl, T. L. Gilliland, D. O. Jobe, J. S. McGurn, J. F. Seamen, J. A. Torres, M. F. Vargas and the Saturn and $\mathrm{Z}$ crews for their dedicated technical support; W. Beezhold, D. L. Cook, G. Cooperstein (NRL), J. Davis (NRL), M. E. Jones (LANL), R. J. Leeper, J. E. Maenchen, M. K. Matzen, D. H. McDaniel, J. P. Quintenz, J. J. Ramirez, A. Toor, and G. Yonas for their vigorous programmatic support that has made this research possible; and M. K. Matzen and J. S. De Groot (UC Davis) for carefully reviewing the manuscript.

\section{REFERENCES}

[1] T. W. L. Sanford, G. O. Allshouse, B. M. Marder, T. J. Nash, R. C. Mock, R. B. Spielman, J. F. Seamens, J. S. McGurn, D. Jobe, T. L. Gilliland, M. Vargas, K. W. Struve, W. A. Stygar, M. R. Douglas, M. K. Matzen, J. H. Hammer, J. S. De Groot, J. L. Eddleman, D. L. Peterson, D. Mosher, K. G. Whitney, J. W. Thornhill, P. E. Pulsifer, and J. P. Apruzese, Phys. Rev. Lett. 77, 5063 (1996).

[2] M. K. Matzen, Phys. Plasmas 4, 1519 (1997).

[3] Gerold Yonas, "Fusion and the Z Pinch", Scientific American, 279, 40 (Aug. 1998).

[4] D. D. Bloomquist, R. M. Stinnett, D. H. McDaniel, J. R. Lee, A. W. Sharpe, J. A. Halbleib, L. G. Shlitt, P. W. Spence, and P. Corcoran, Proc. 6th Int. IEEE Pulsed Power Conf., Arlington, VA, ed. P. J. Turchi and B. H. Bernstein (Institute of Electrical and Electronic Engineers, New York, 1987), p. 310. 
[5] R. B. Spielman, C. Deeney, G. A. Chandler, M. R. Douglas, D. L. Fehl, M. K. Matzen, D. H. McDaniel, T. J. Nash, J. L. Porter, T. W. L. Sanford, J. F. Seamen, W. A. Stygar, K. W. Struve, S. P. Breeze, J. S. McGurn, J. A. Torres, D. M. Zagar, T. L. Gilliland, D. O. Jobe, J. L. McKenney, R. C. Mock, M. Vargas, T. Wagoner, and D. L. Peterson, Phys. Plasmas 5, 2105 (1998).

[6] J. L. Porter, Am. Phys. Soc. 44, 1948 (1997).

[7] M. K. Matzen, C. Deeney, R. J. Leeper, J. L. Porter, R. B. Spielman, G. A. Chandler, M. S. Derzon, M. R. Douglas, D. L. Fehl, D. E. Hebron, T. J. Nash, R. E. Olson, L. E. Rugglers, T, W. L. Sanford, J. F. Seamen, K. W. Struve, W. A. Stygar, and D. L. Peterson, "Fast Z-Pinches as a Dense Plasma, Intense X-Ray Source for Plasma Physics and Fusion Applications", accepted for publication in Plasma Physics and Controlled Fusion (1998).

[8] R.J. Leeper, T.E. Alberts, J.R. Asay, P.M. Baca, K.L. Baker, S.P. Breeze, G.A. Chandler, D.L. Cook, G.W. Cooper, C. Deeney, M.S. Derzon, M.R. Douglas, D.L. Fehl, T. Gilliland, D.E. Hebron, M.J. Hurst, D.O. Jobe, J.W. Kellogg, J.S. Lash, S.E. Lazier, M.K. Matzen, T.A. Mehlhorn, D.H. McDaniel, J.S. McGurn, A.R. Moats, R.C. Mock, D.J. Muron, T.J. Nash, R.E. Olson, J.L. Porter, J.P. Quintenz, P.V. Reyes, L.E. Ruggles, C.L, Ruiz, F.A. Schmidlapp, J.F. Seamen, R.B. Spielman, T.W.L. Sanford, M.A. Stark, K.W. Struve, W.A. Stygar, D.R. TibbettsRussell, J.A. Torres, M. Vargas, T.C. Wagoner, C. Wakefield, J.H. Hammer, D.D. Ryutov, M. Tabak, S.C. Wilks, R.L. Bowers, K.D. McLenithan, and D.L. Peterson, "Z-pinch Driven Inertial Confinement Fusion Target Physics Research at Sandia National Laboratoies", proceedings 17th IAEA Fusion Energy Conference, Yokohama, Japan, Oct. 19-24, 1998.

[9] R. E. Olson, J. L. Porter, G. A. Chandler, D. L. Fehl, D. O. Jobe, R. J. Leeper, M. K. Matzen, J. S. McGurn, D. D. Noack, L. E. Ruggles, P. Sawyer, J. A. Torres, M. Vargas, D. M. Zagar, H. N. Kornblum, T. J. Orzechowski, D. D. Phillion, L. J. Suter, A. R. Thiessen, and R. J. Wallace, Phys. Plasmas 4, 1818 (1997).

[10] R.E. Olson, G.A. Chandler, M.S. Derzon, D.E. Hebron, J.S. Lash, R.J. Leeper, T.J. Nash, G.E. Rochau, T.W.L. Sanford, N.B. Alexander, and C.R. Gibson, "Indirect-Drive ICF Target Concepts for the X-1 Z-Pinch Facility", to be published in Fusion Technology (March 1999).

[11] T. W. L. Sanford, R. C. Mock, T. J. Nash, W. G. Whitney, P. E. Pulsifer, J. P. Apruzese, D. Mosher, D. L. Peterson, and M. G. Haines, "Systematic Trends in X-Ray Emission Characteristics of Variable-Wire-Number, Fixed-Mass, Aluminum-Array, Z-Pinch Implosions," submitted to Phys. Plasmas (1998).

[12] T. W. L. Sanford, R. C. Mock, R. B. Spielman, D. L. Peterson, D. Mosher, and N. F. Roderick, Phys. Plasmas 5, 1337 (1998).

[13] T. W. L. Sanford, R. C. Mock, R. B. Spielman, D. L. Peterson, D. Mosher, and N. F. Roderick, Phys. Plasmas 5, 1355 (1998).

[14] B.M. Marder, Math. of Comp., 29, 434 (1973). 
[15] B. M. Marder, T. W. L. Sanford, and G. O. Allshouse, Phys. Plasmas, 5, 2997 (1998).

[16] J. P. Chittenden, R. Aliaga Rossel, S. V. Lebedev, I. H. Mitchell, M. Tatarakis, A. R. Bell, and M. G. Haines, Phys. Plasmas 4, 4309 (1997).

[17] J. P. Chittenden, et al, work in progress.

[18] Y.T. Lee and R.M. More, Phys. Fluids 27, 1273 (1984).

[19] R.M. More, K.H. Warren, D.A. Young, and G.B. Zimmerman, Phys. Fluids 31, 3059 (1988).

[20] D. H. Kalantar and D. A. Hammer, Phys. Rev. Lett. 71, 3806 (1993).

[21] T.A. Shelkovenko, S.A. Pikuz, A.R. Mingaleev, and D.A. Hammer, Rev. Sci. Instrum., to be published Jan. 1999.

[22] M. G. Haines, IEEE Trans. Plasma Sci., 26, 1275 (1998).

[23] D. L. Peterson, R. L. Bowers, J. H. Brownell, A. E. Greene, K. D. McLenithan, T. A. Oliphant, N. F. Roderick, and A. J. Scannapieco, Phys. Plasmas 3, 368 (1996).

[24] D. L. Peterson, R. L. Bowers, J. H. Brownell, C. Lund, W. Matuska, K. McLenithan, H. Oona, C. Deeney, M. Derzon, R. B. Spielman, T. J. Nash, G. Chandler, R. C. Mock, T. W. L. Sanford, and M. K. Matzen, Dense Z-Pinches: Fourth International Conference, edited by N. R. Pereira, J. Davis, and P. E. Pulsifer, 1997 The American Institute of Physics 1-56396-610-7/97, p 201-210.

[25] T. W. L. Sanford, T. J. Nash, R. C. Mock, R. B. Spielman, K. W. Struve, J. H. Hammer, J. S. De Groot, K. G. Whitney, and J. P. Apruzese, Phys. Plasmas 4, 2188 (1997).

[26] C. Deeney, P. D. Pell, B. H. Failor, S. L. Wong, J. P. Apruzese, K. G. Whitney, J. W. Thornhill, J. Davis, E. Yadlowsky, R. C. Hazelton, J. J. Moschella, T. Nash, and N. Loter, Phys. Rev. E 51, 4823 (1995).

[27] S. V. Lebedev, I. H. Mitchell, R. Aliaga-Rossel, S. N. Bland, J. P. Chittenden, A. E. Dangor, and M. G. Haines, "Azimuthal Structure and Global Instability In the Implosion Phase of Wire Array Z-Pinch Experiments" submitted to Phys. Rev. Lett . (1998).

[28] F. N. Beg, A. E. Dangor, P. Lee, M. Tatarakis, S. L. Niffikeer, and M. G. Haines, Plasma Phys. Fusion 39, 1 (1997).

[29] D. Mosher and D. Colombant, Phys. Rev. Lett. 68, 2600(1992).

[30] D. Mosher, BEAMS'94, Proceedings of 10th International Conference on High power Particle Beams, NTIS PB95-1443417, pp 159-162. 
[31] R. B. Spielman, J. S. De Groot, T. J. Nash, J. McGurn, L. Ruggles, M. Vargas, and K. G. Estabrook, Dense z-Pinches (3rd International Conference, London, UK, 1993), AIP Conference Proceedings 299, editors H. Haines and A. Knight, American Institute of Physics, New York 1994, L.C. Catalog Card No. 93-74569, pp. 404-420.

[32] M. S. Derzon, G. O. Allshouse, G. A. Chandler, et al., Bull. Am. Phys. Soc. 42, 1994, (1997).

[33] T.J. Nash, M.S. Derzon, G.A. Chandler, R.J. Leeper, D.L. Fehl, J.F. Seaman, J. McGurn, S Lazier, J. Torres, D. Jobe, T. Gilland, M. Hurst, R. Mock, J McKenney, D. Hebron, T. Alberts, J.J. MacFarlane, D. L. Peterson, R. Bowers, W, Matuska, and D.D. Ryutov, "High Temperature Dynamic Hohlraums on the Pulsed Power Driver Z", submitted to Phys. Plasmas (Nov. 1998).

[34] M. P. Desjarlais and B. M. Marder, "Theory of Wire Number Scaling in Wire-Array Z Pinches", submitted to Phys. Plasmas (Nov. 1998).

[35] D.B. Reisman, Ph.D. Dissertation, University of California, Davis, 1998.

[36] J.L. Eddleman, J.H. Hammer, and C.W. Hartman, Numerical Modeling of the Race Experiment, Bull. Am. Phys. Soc. 32: 1912, 1987.

[37] T. W. L. Sanford, R. B. Spielman, G. O. Allshouse, et al., IEEE Trans. Plasma Sci., 26, 1086 (1998).

[38] T. W. L. Sanford, R. C. Mock, B. M. Marder, M. P. Desjarlais, T. J. Nash, R. B. Spielman, D. L. Peterson, K. G. Whitney, J. P. Apruzese, P. E. Pulsifer, and D. Mosher, "Wire Array ZPinch Insights for High X-Ray Power Generation", to be published in the Proceedings of the $12^{\text {th }}$ International Conference on High-Power Particle Beams: BEAMS'98 (Haifa, Israel, June 7-12, 1998).

[39] J.P. Chittenden, I. H. Mitchell, A. R. Bell, R. Aliaga-Rossel, J. M. Bayley, F. N. Beg, A. Lorenz, M. Tatarakis, A. E. Dangor, M. G. Haines, Bull. Am. Phys. Soc. 41, 1471(1996).

[40] J. H. Hammer, J. L. Eddleman, P. T. Springer, M. Tabak, A. Toor, K. L. Wong, G. B. Zimmerman, C. Deeney, R. Humphreys, T. J. Nash, T. W. L. Sanford, R. B. Spielman, and J. S. De Groot, Phys. Plasmas 3, 2063 (1996).

[41] T. W. L. Sanford, R. C Mock, B. M. Marder, T. J. Nash, R. B. Spielman, D. L. Peterson, N. F. Roderick, J. H. Hammer, J. S. De Groot, D. Mosher, K. G. Whitney, and J. P. Apruzese, Sandia National Laboratories Technical Report, SAND96-3031 (July 1997) and Dense Z-

Pinches: 4th International Conference (Vancouver, Canada, May 28-30, 1997), Edited by N.R. Pereira, J. Davis, and P.E. Pulsifer, The American Institute of Physics, 1-56396-610-7197, pp. 561-573. 
[42] C. Deeney, T. J. Nash, R. B. Spielman, J. F. Seaman, M. R. Douglas, J. S. McGurn, D. O. Jobe, T. L. Gilliland, R. C. Mock, K. W. Struve, K. G. Whitney, P. E. Pulsifer, J. P. Apruzese, and J. W. Thornhill, Phys. Plasma 5, 2431 (1998) and references therein.

\section{FIGURE CAPTIONS}

1. (A) Peak total radiated power (solid line) and pulsewidth, (B) Radial convergence, (C) Total radiated energy normalized by calculated kinetic energy assuming a 10:1 convergence, (D) Peak total radiated power $(A)$ divided by the normalizied energy plotted in (C) (solid line) and the pulsewidth of the 4-to-6 keV x-ray channel (dashed line). and $\square$ correspond to the 8.6- and 12-mm initial-radius data, repectively.

2. Normalized pulseshape in three energy channels for the large-radius (12-mm initial radius) geometry where (A) $\mathrm{G}=5.5 \mathrm{~mm}$ and (B) $\mathrm{G}=0.4 \mathrm{~mm}$. Time is measured from a linear extrapolation of the leading edge of the main current pulse to the abscissa. (C) and (D) are associated time-integrated $\mathrm{x}$-ray images, respectively.

3. (A) Lineouts of axially-integrated $x$-ray images as a function of time (upper right-hand corner), for a 1.3-mm interwire gap array. (B) Image at -19 ns. (C) Normalized total radiated power pulse. Zero time corresponds to peak radiated power. The wire array geometry was identical to that of the small-radius array, except 24 wires were mounted at a radius of only 5 $\mathrm{mm}$. 
4. TIP $x-y$ density contours for a small-radius load (having 10 current return posts) at stagnation for (A) a 10-wire array in the plasma-wire regime and (B) a 20-wire array in the plasma-shell regime.

5. (A) Comparison of the $\mathrm{MH} 2 \mathrm{D}$ density of a single $15-\mu \mathrm{m}$ diameter wire as a function of radius with the normalizied experimental line density measured (at two different axial locations on the same $13-\mu \mathrm{m}$ diameter wire), both at the end of the idealizied current prepulse for a 64-wire array. The (uncalibrated) units for line densities are each normalized to the MH2D core density. (B) MH2D ion temperature, electron temperature, ionization state associated with (A).

6. MH2D electron-number density as a function of time at a radius corresponding to $\mathrm{G} / 2$, for an equivalent wire in a wire array having $1630-\mu \mathrm{m}, 2425-\mu \mathrm{m}, 4817.5-\mu \mathrm{m}, 6415-\mu \mathrm{m}$, and $13610-$ $\mu \mathrm{m}$ diameter wires. Zero time corresponds to the start of the main current pulse.

7. MH2D mass-density log contours calculated in the $r-z$ plane for a single aluminum wire, 30 ns into the main current pulse (A) for one of $24,25-\mu \mathrm{m}$ wires and (B) for one of $64,15-\mu \mathrm{m}$ wires. MH3D mass-density log contours calculated in the $x-y$ plane, 35 ns into the main current pulse (D) for $16,30-\mu \mathrm{m}$ wire, and (E) a $48,17.5-\mu \mathrm{m}$ wire array, both having the small-radius geometry.

8. (A) $t_{m} / t_{p}$ and $a_{m} / r_{0}$ as a function of gap in the Heuristic Model, illustrating the position of the critical wire number and gap, for the small-radius load. (B) Heuristic Model components and comparison with the measured risetime, for the small-radius load. 
9. Left: Tungsten-wire-plasma Schleiren image. Right: Carbon-fiber MHD simulation from Chittenden, et al. [39] at three times during development.

10. Measured (A) $x$-ray power in indicated energy channels, (B) diameter of $x$-ray image radial lineouts averaged over $z$ axis, $(C)$ electron core temperaure from free-to-bound $x$-ray continuum, and (D) ion velocity from $\mathrm{K}$-shell line Doppler shifts, all for the small-radius load having a gap of $0.6 \mathrm{~mm}$ using 90 wires. E-RMHC:R simulations of the measured power and diameter are compared in (A) and (B).

11. Measured and E-RMHC simulated (A) total-radiated-power pulsewidth and (B) total radiated peak power as a function of mass, for the $\sim 192-$ wire, $\mathrm{r}_{0}=12-\mathrm{mm}$ Saturn mass scan. Measured (C) total-radiated-power pulsewidth and (D) total radiated peak power relative to the measured power at the insulator stack as a function of mass for the $\sim 120$-wire, $\mathrm{r}_{0}=10-\mathrm{mm} \mathrm{Z}$ mass scan. Measured and E-RMHC simulated (E) total-radiated-power pulsewidth and (F) total radiated peak power as a function of array radius, for the $\sim 136$-wire, mass $=0.6-\mathrm{mg}$ load Saturn radius scan.

12. E-RMHC shell velocity (dashed curve), and shell thickness (dotted curve) just as stagnation begins, including the associated width of the resulting radiation pulse (solid curve) as a function of (A) mass and (B) radius, for the Saturn mass and radius scan, respectively. 

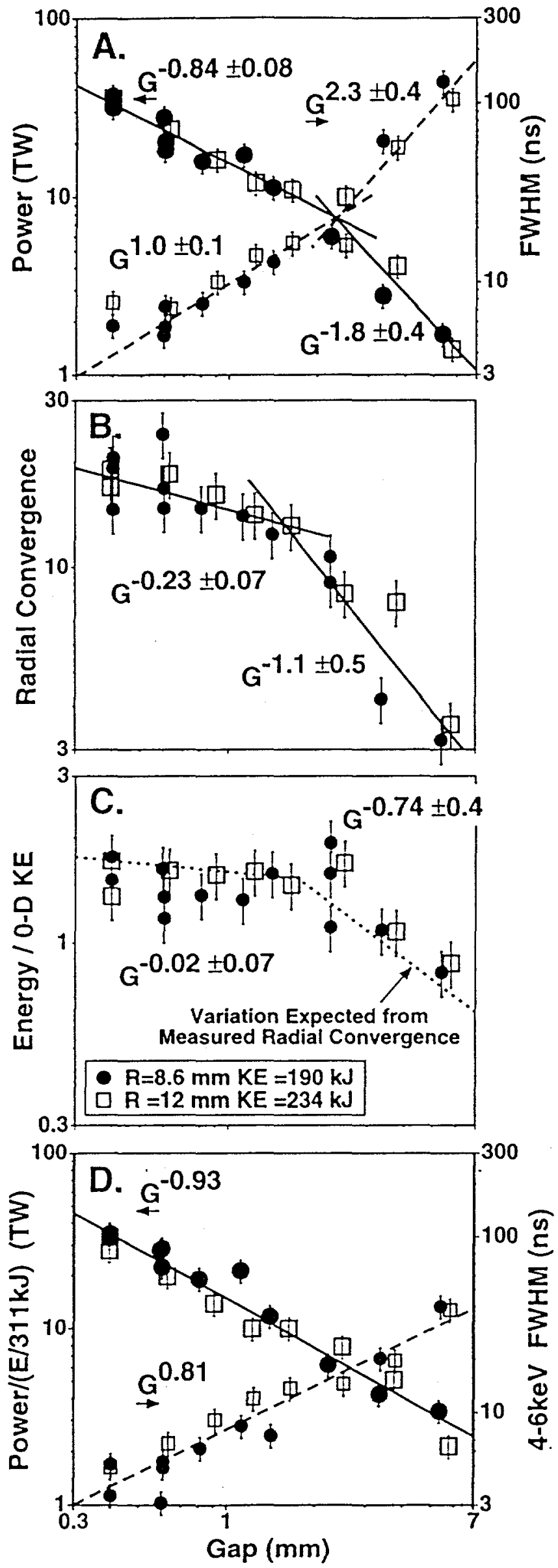

Fis. 1 

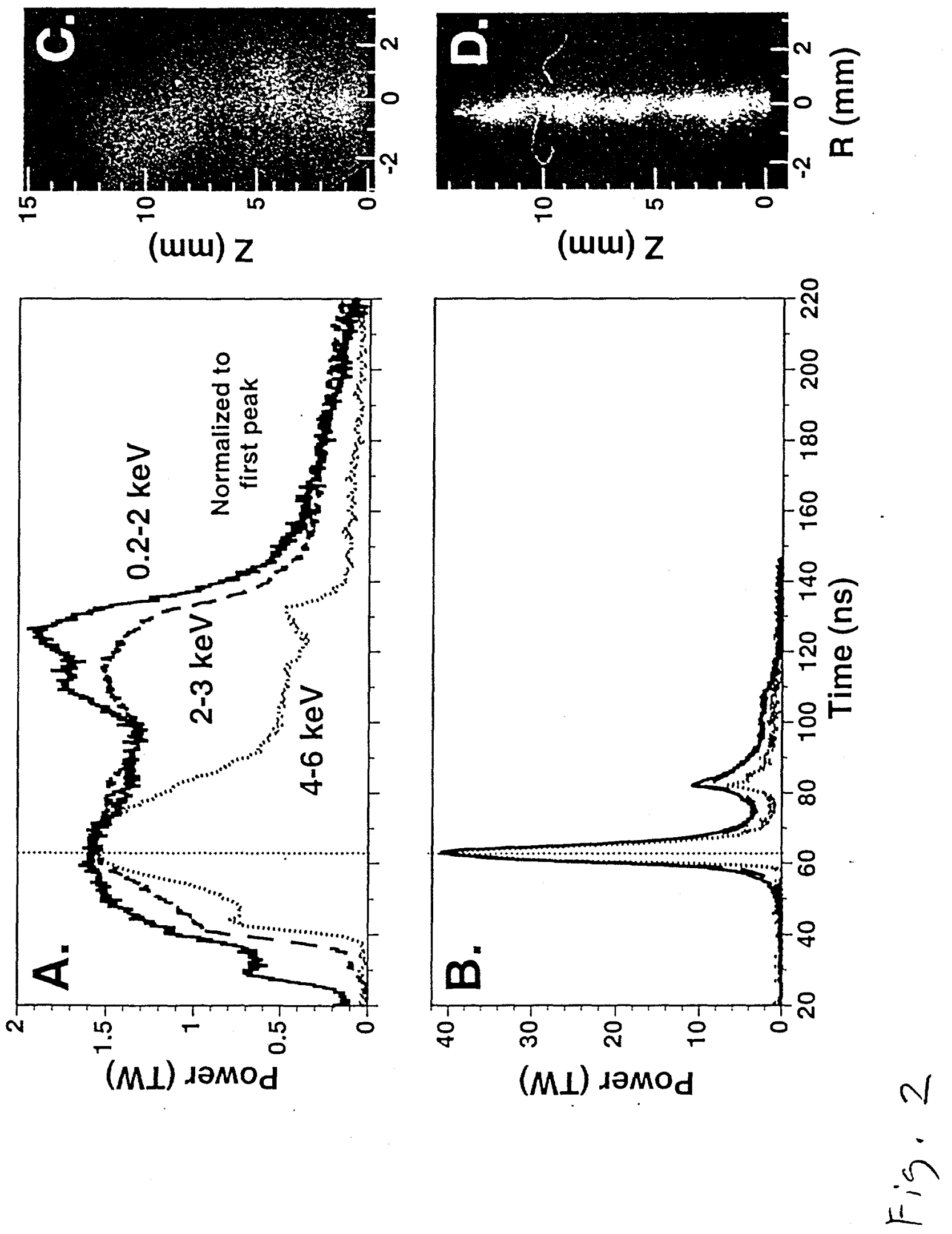

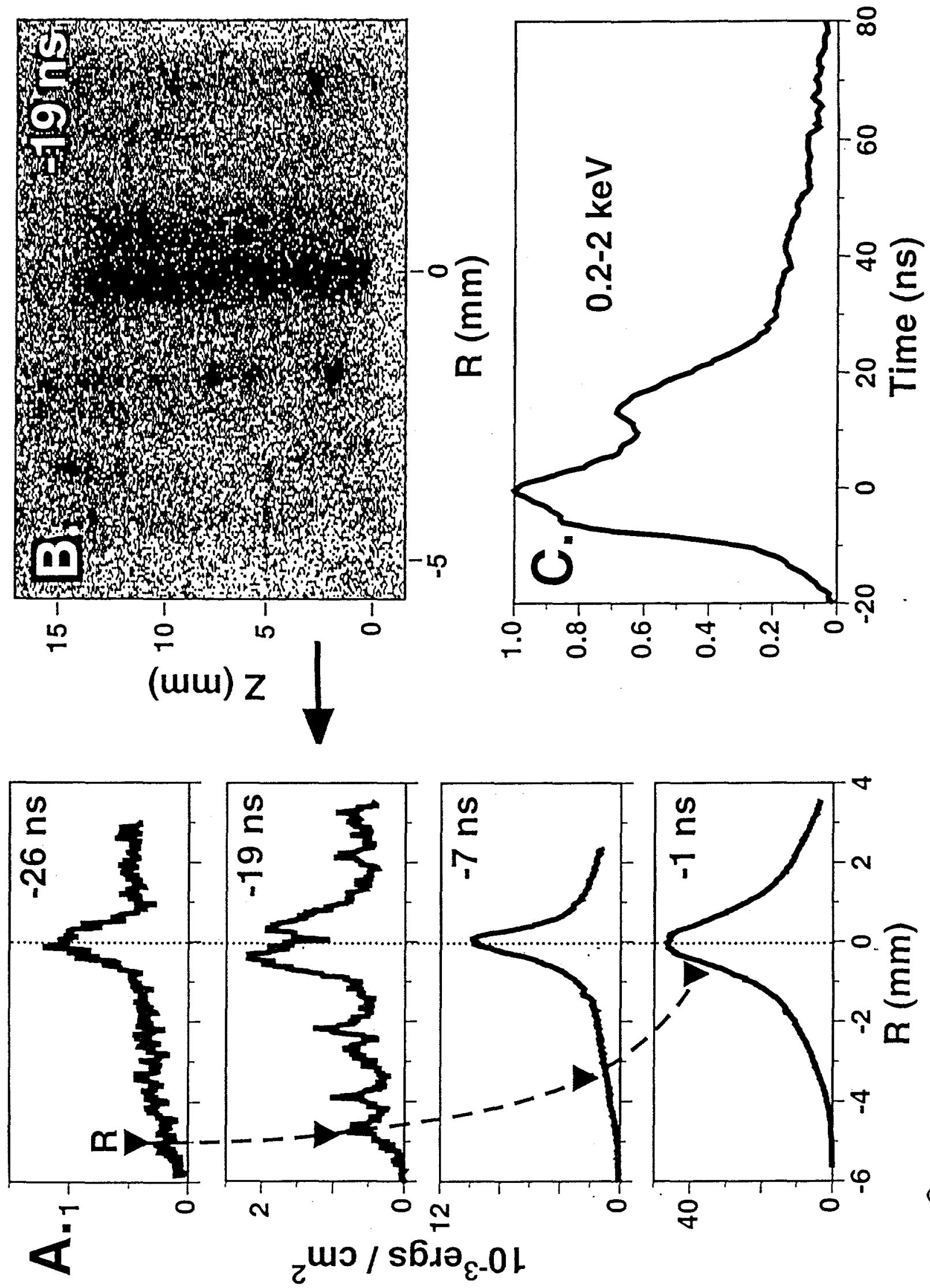


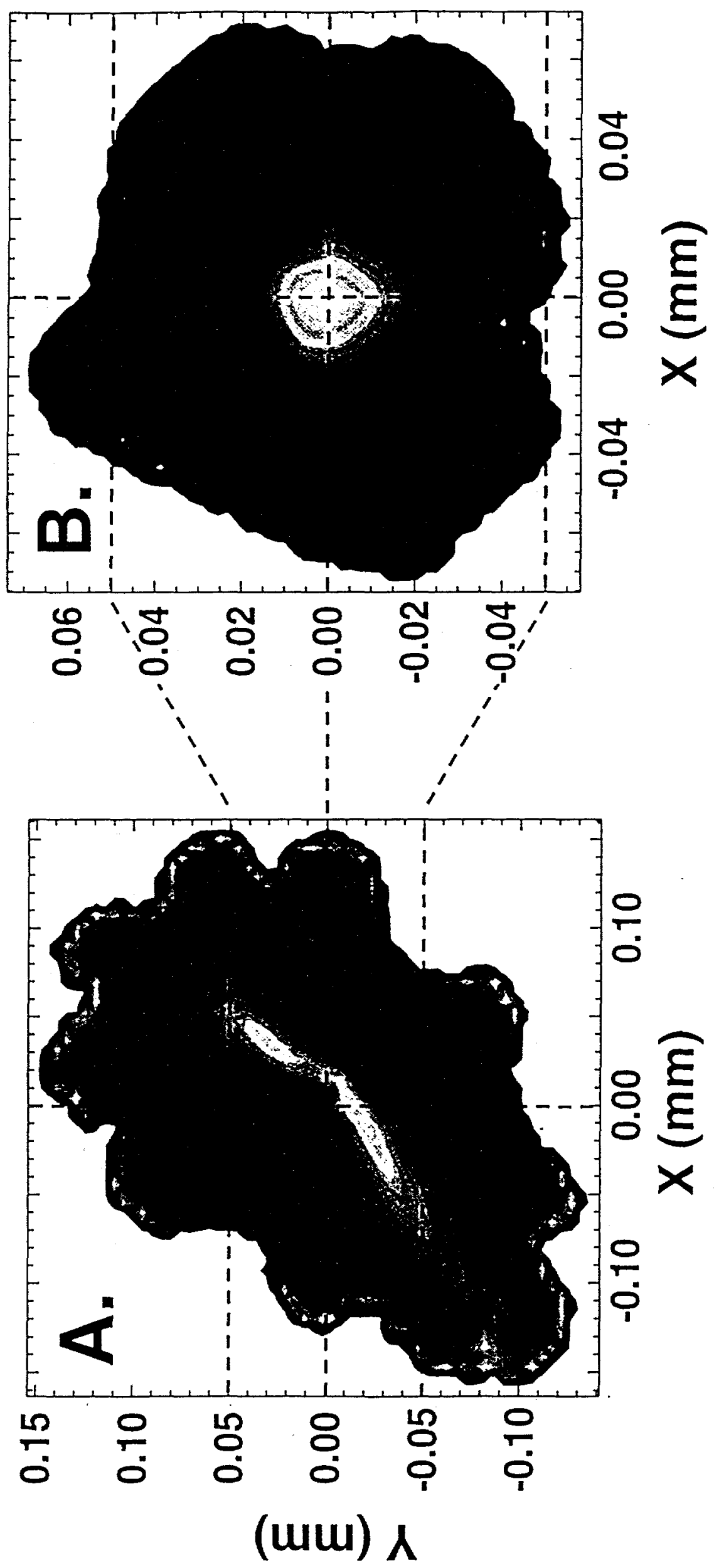

J in 

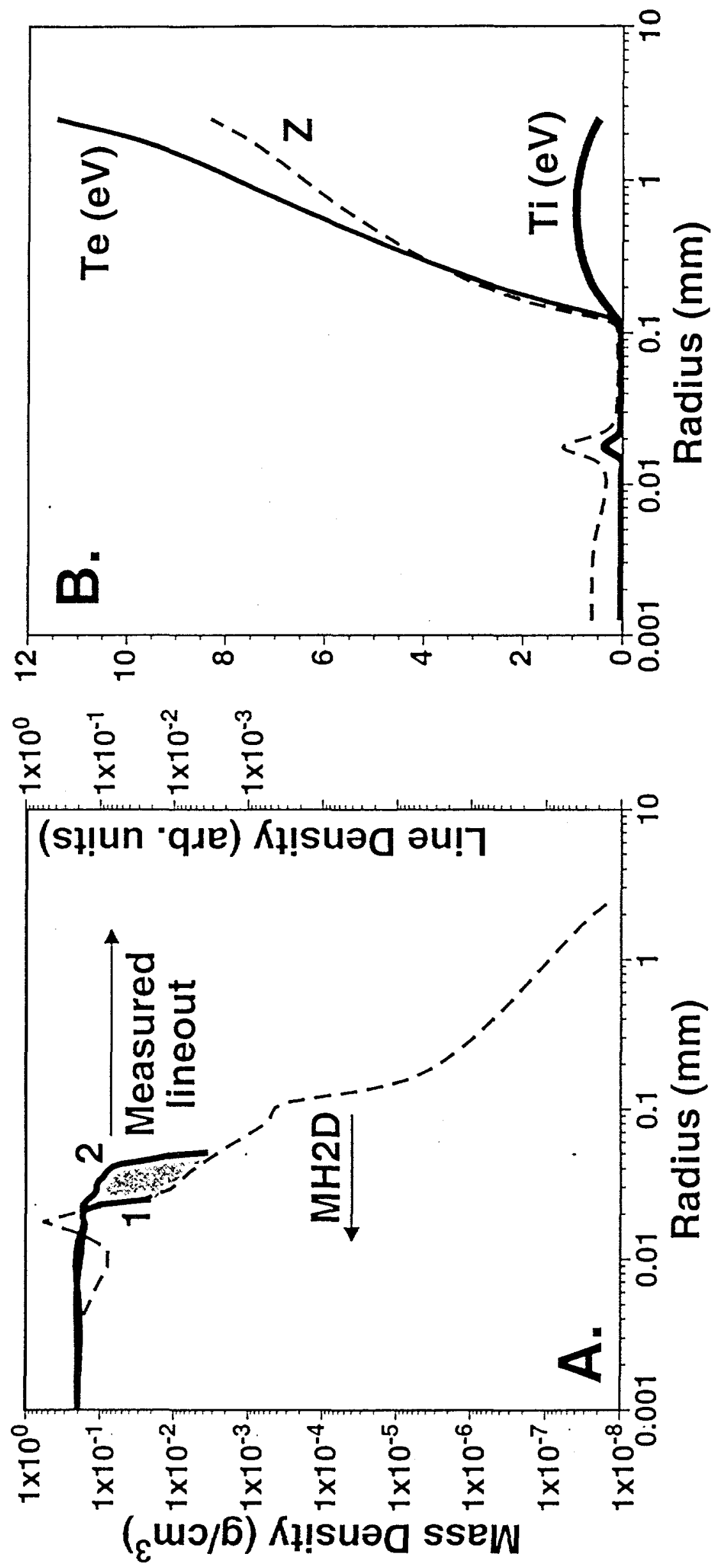


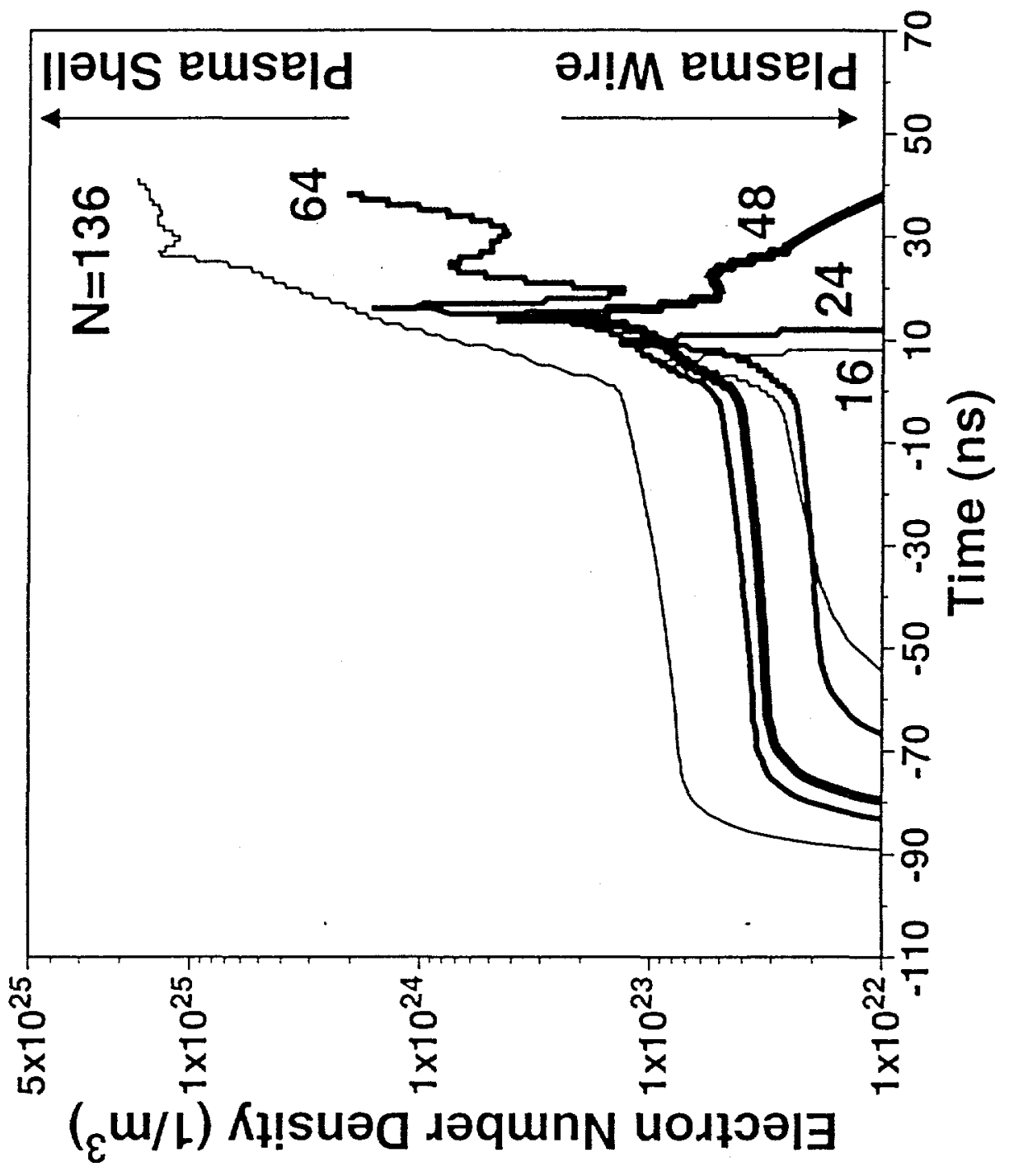




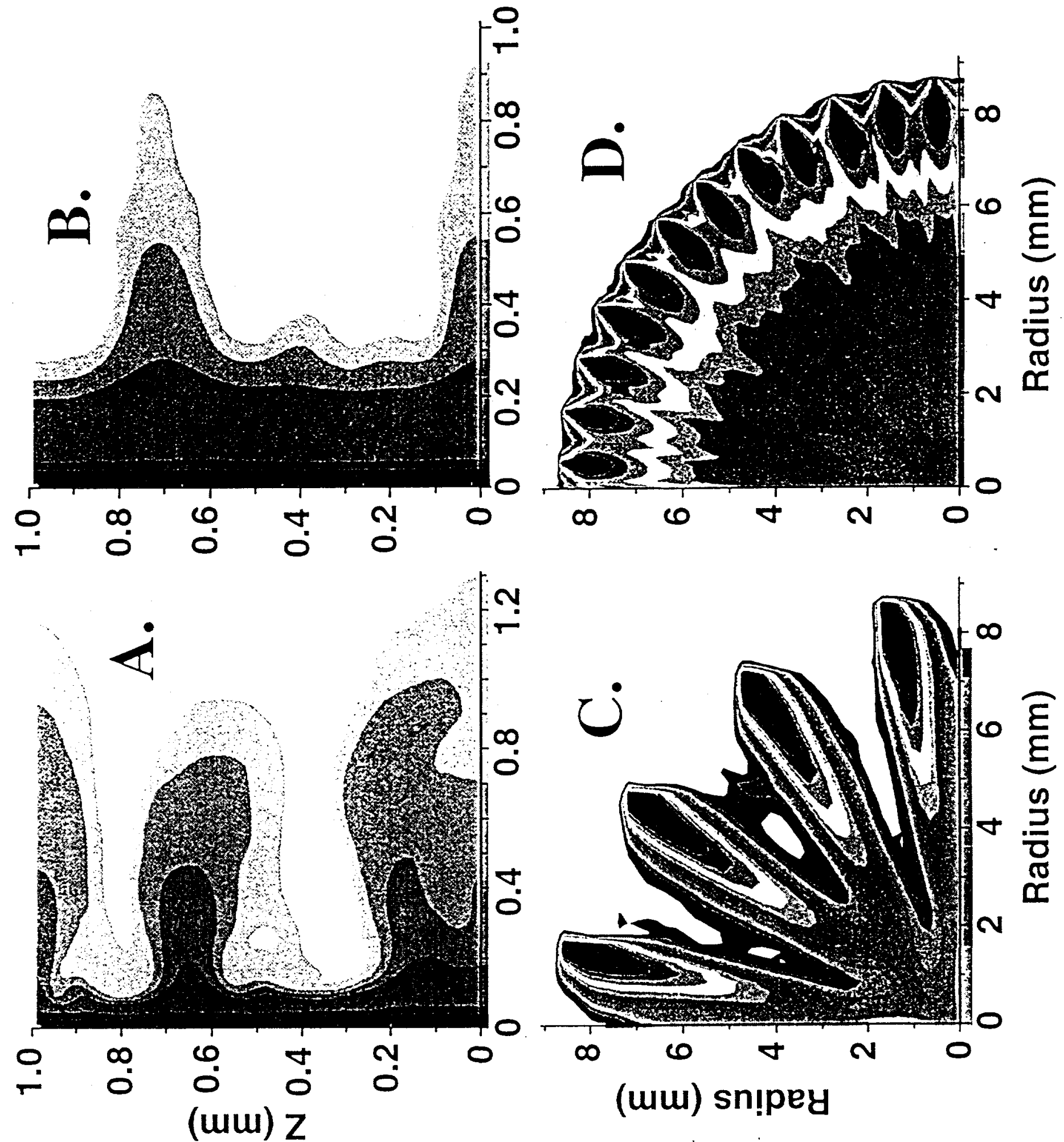



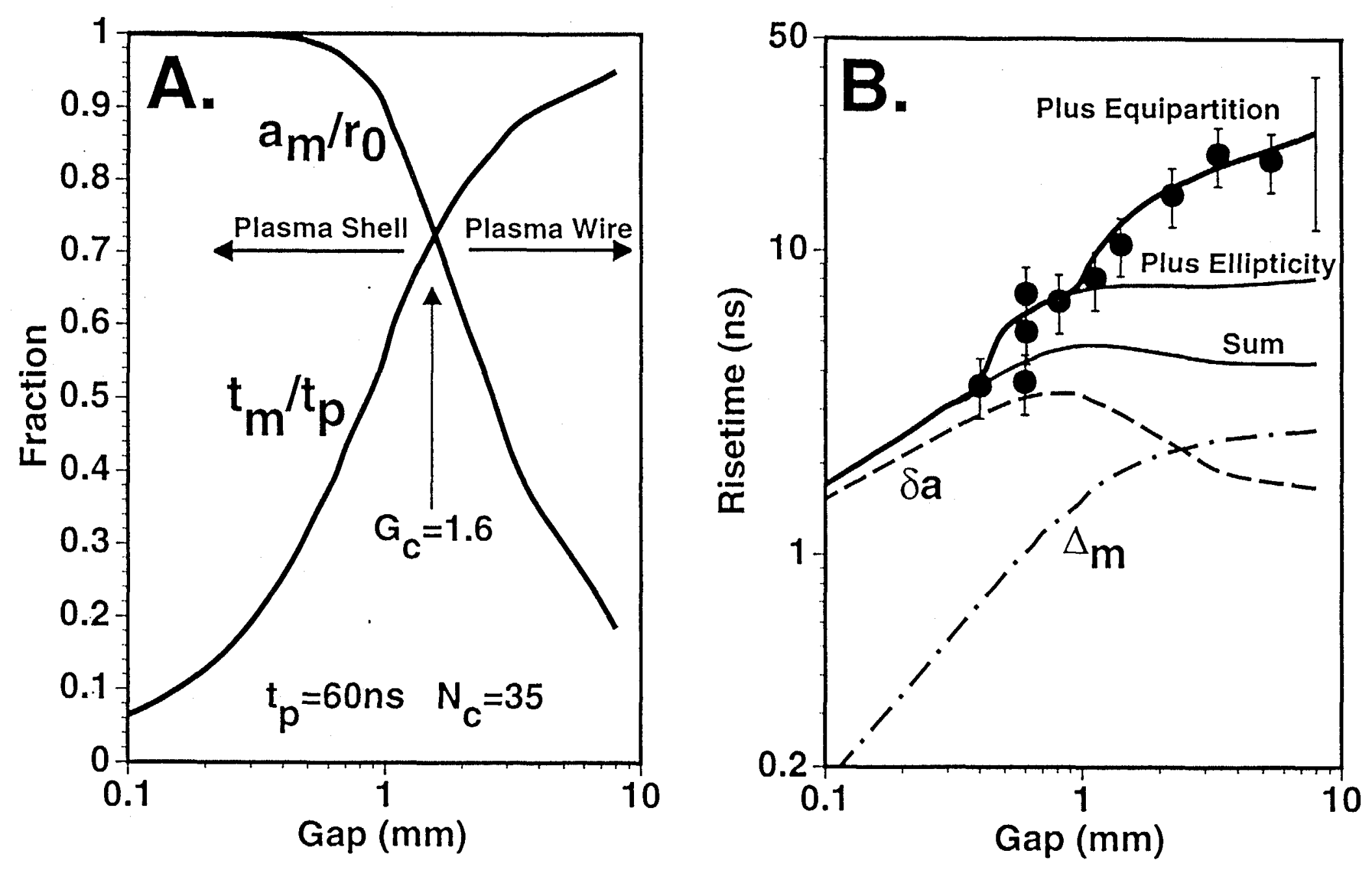

Fig. 8 

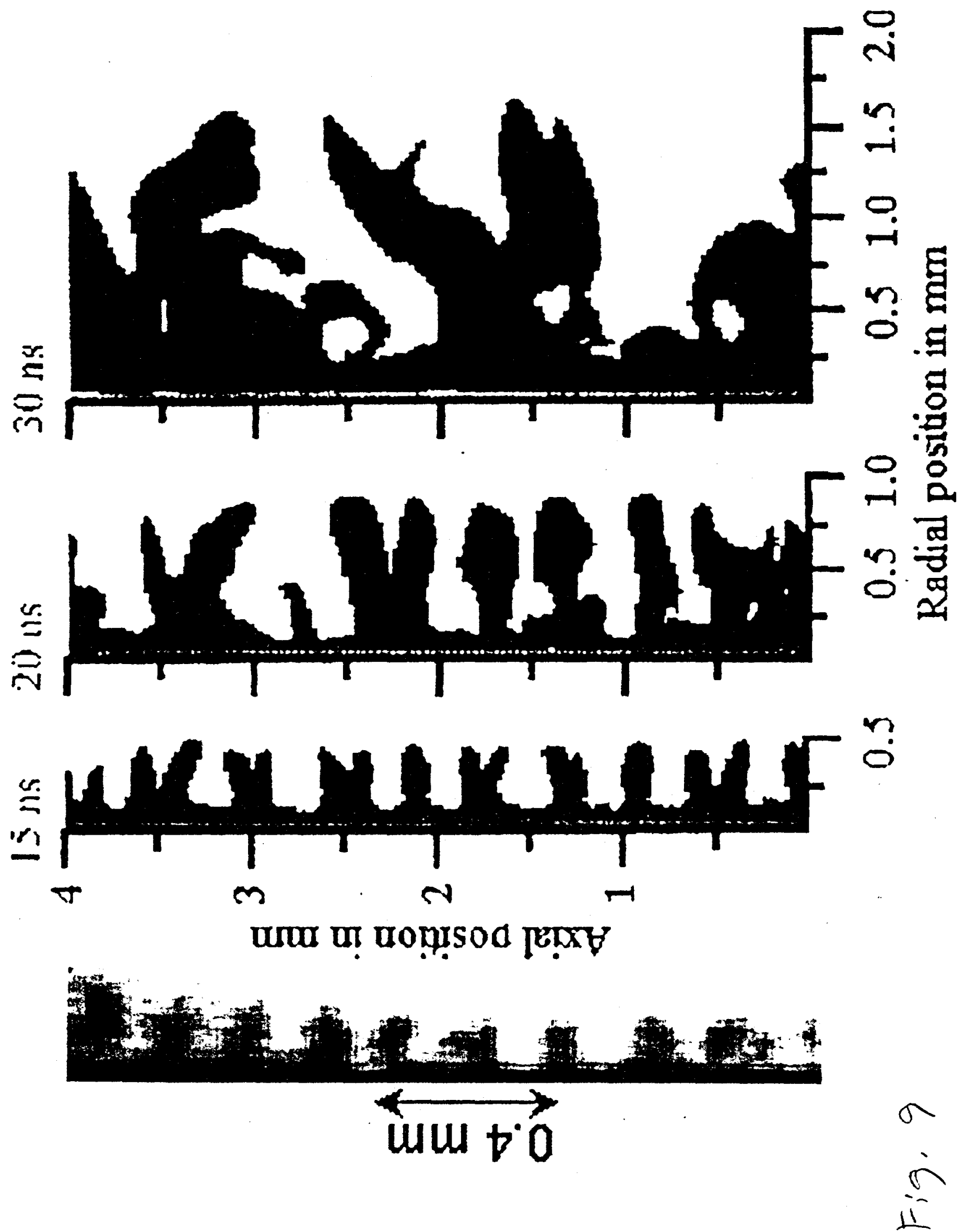

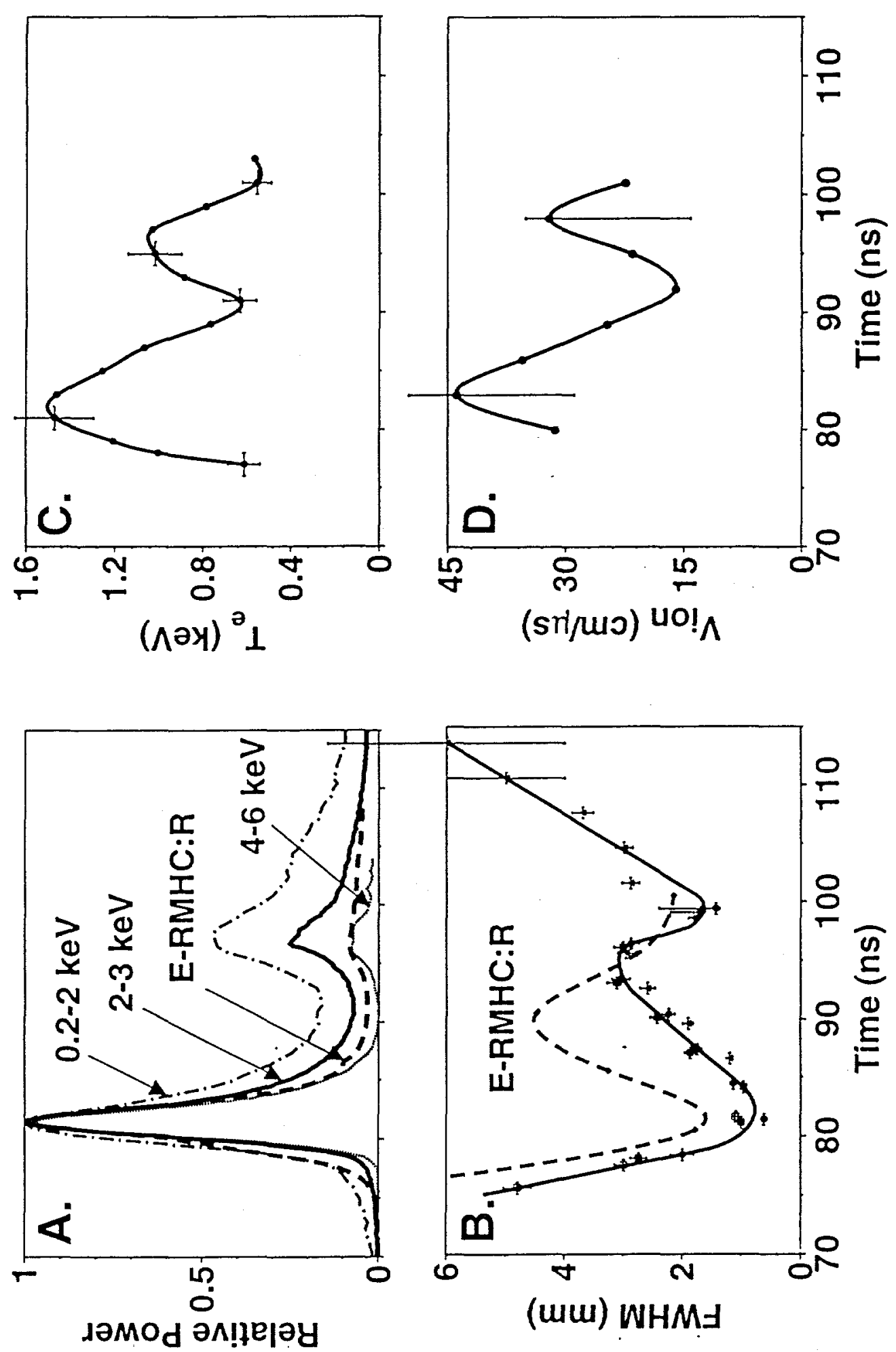

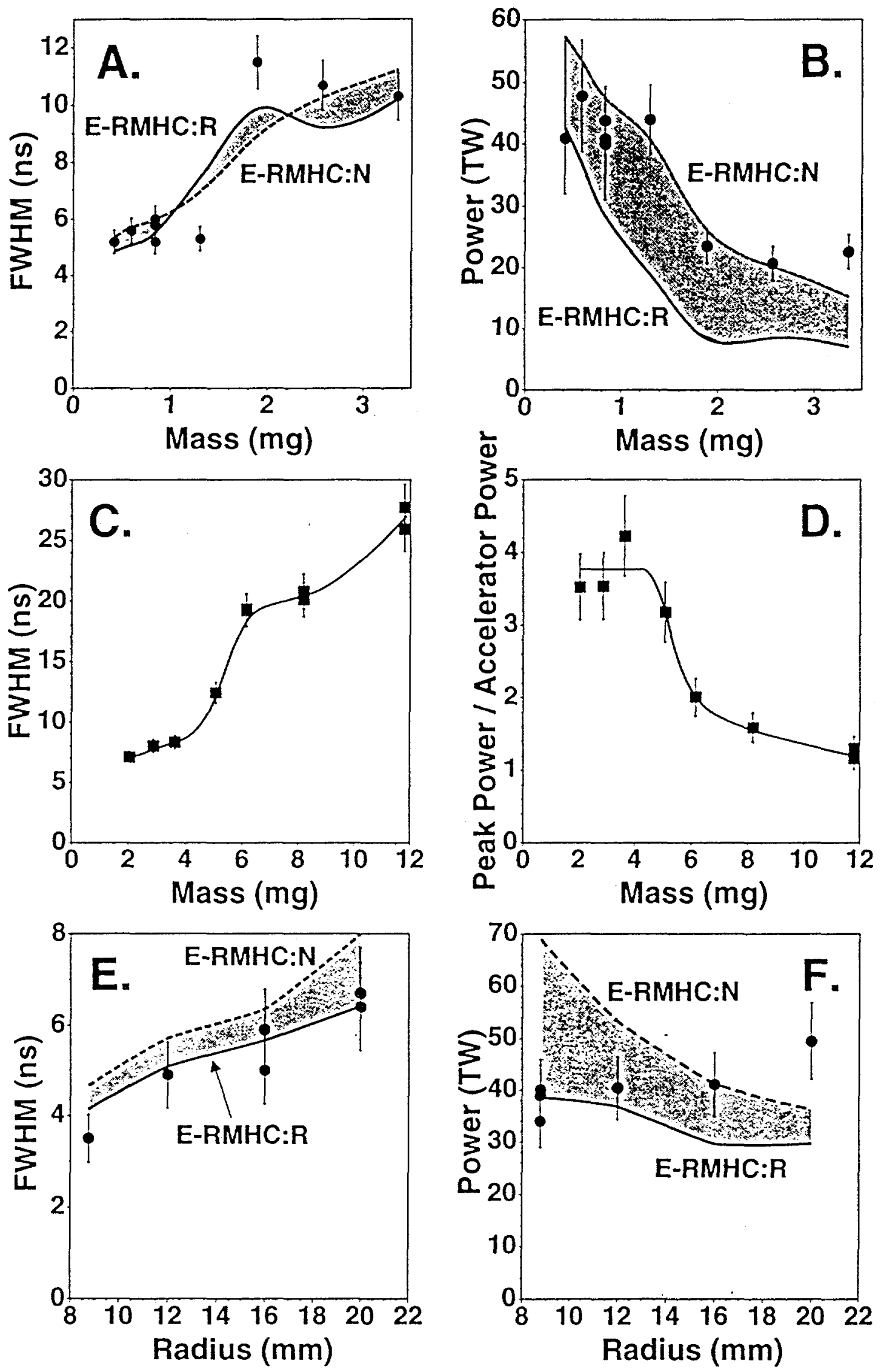
(su/mw) К뀌잇
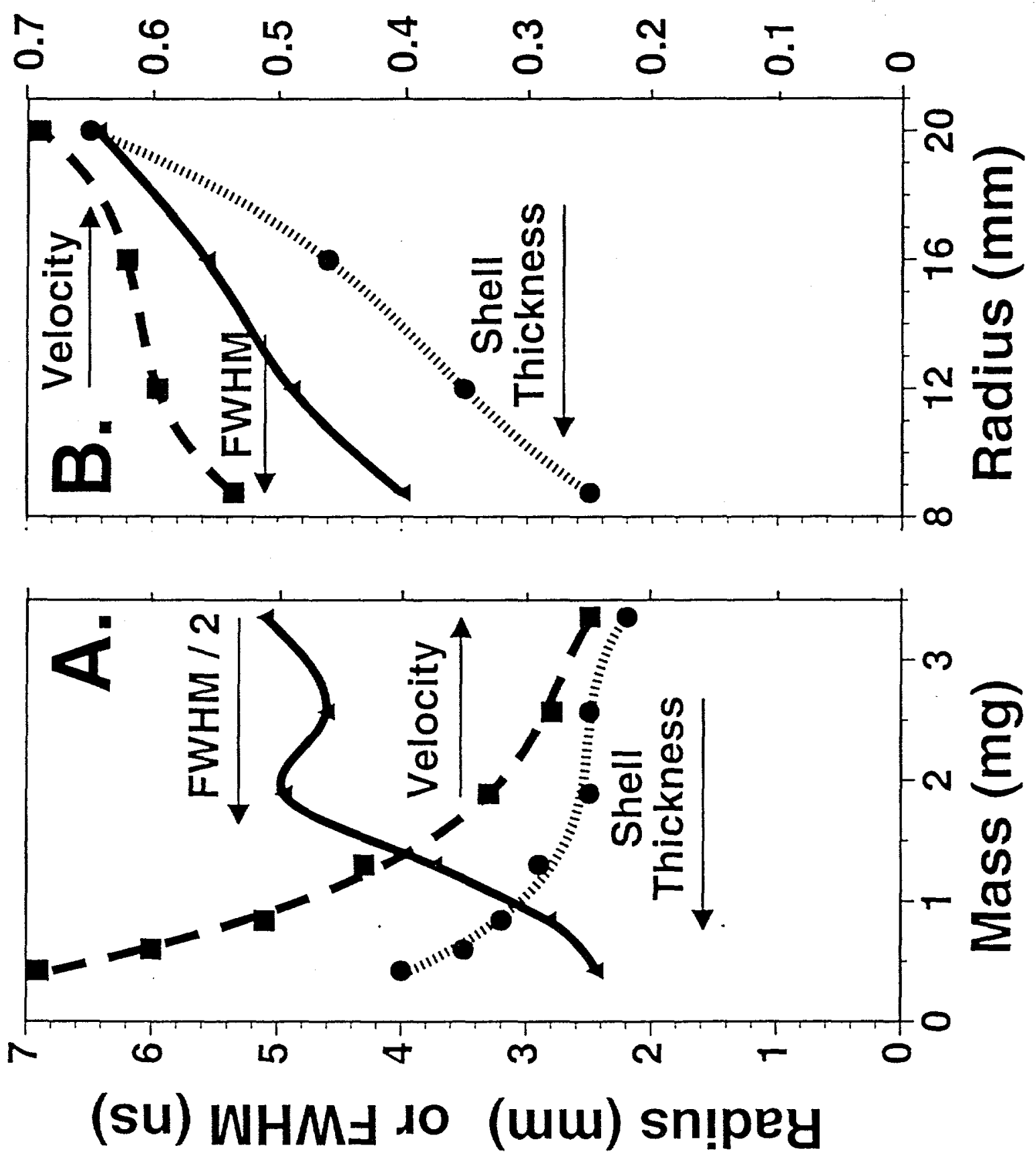